\title{
A distinct molecular profile associated with mucinous epithelial ovarian cancer
}

\author{
VA Heinzelmann-Schwarz',2, M Gardiner-Garden', SM Henshall', JP Scurry ${ }^{3}$, RA Scolyer ${ }^{4}$, AN Smith', A Bali', \\ P Vanden Bergh', S Baron-Hay', C Scott ${ }^{5}$, D Fink', NF Hacker', RL Sutherland' and PM O'Brien ${ }^{*}, 1$ \\ 'Cancer Research Program, Garvan Institute of Medical Research, Darlinghurst, NSW 20 I0, Australia; ${ }^{2}$ Division of Gynecology, University Hospital Zurich, \\ Switzerland; ${ }^{3}$ South Eastern Area Laboratory Service, Prince of Wales Hospital, Randwick, NSW 2031, Australia; ${ }^{4}$ Department of Anatomical Pathology, \\ Royal Prince Alfred Hospital, Camperdown, NSW 2050, Australia; ${ }^{5}$ Kolling Institute of Medical Research, Royal North Shore Hospital, St Leonards, NSW \\ 2065, Australia; ${ }^{6}$ Gynaecological Cancer Centre, Royal Hospital for Women, Randwick, NSW 203I, Australia
}

\begin{abstract}
Mucinous epithelial ovarian cancers (MOC) are clinically and morphologically distinct from the other histological subtypes of ovarian cancer. To determine the genetic basis of MOC and to identify potential tumour markers, gene expression profiling of 49 primary ovarian cancers of different histological subtypes was performed using a customised oligonucleotide microarray containing $>59000$ probesets. The results show that MOC express a genetic profile that both differs and overlaps with other subtypes of epithelial ovarian cancer. Concordant with its histological phenotype, MOC express genes characteristic of mucinous carcinomas of varying epithelial origin, including intestinal carcinomas. Differences in gene expression between MOC and other histological subtypes of ovarian cancer were confirmed by RT-PCR and/or immunohistochemistry. In particular, galectin 4 (LGALS4) was highly and specifically expressed in MOC, but expressed at lower levels in benign mucinous cysts and borderline (atypical proliferative) tumours, supporting a malignant progression model of MOC. Hence LGALS4 may have application as an early and differential diagnostic marker of $\mathrm{MOC}$.

British Journal of Cancer (2006) 94, 904-913. doi:10.1038/sj.bjc.6603003 www.bjcancer.com

Published online 28 February 2006

(c) 2006 Cancer Research UK
\end{abstract}

Keywords: ovarian cancer; mucinous; microarray; immunohistochemistry; diagnosis

Carcinomas arising from the epithelial cells of the ovary are the fifth most common malignancy in women and the leading cause of death from gynaecological cancers. Epithelial ovarian cancers comprise a group of related but distinct carcinomas that likely arise from a common epithelial cell type but develop via differentiation pathways and differ in their clinical presentation and aetiology. They are currently classified into different histological subtypes (including serous, endometrioid, mucinous and clear cell) based on their morphological resemblance to normal epithelia in the gynaecological and intestinal tracts; however the genetic basis underlying their divergence is poorly understood.

The majority of mucinous ovarian cancers (MOC) are diagnosed at an early stage, either as borderline (atypical proliferative) tumours or low-grade carcinomas, and have an excellent prognosis (Sherman et al, 2004). Although less common, advanced MOC is associated with a very poor survival that surpasses the poor prognosis for women with advanced stage serous ovarian cancer (Sherman et al, 2004). Accumulating pathological and epidemiological evidence supports a progression model of MOC, from benign cysts to borderline tumours to invasive adenocarcinoma (Feeley and Wells, 2001; Shih and Kurman, 2004). However, it can

*Correspondence: Dr PM O'Brien; E-mail: p.obrien@garvan.org.au Received 30 August 2005; revised 5 January 2006; accepted 17 January 2006; published online 28 February 2006 be difficult to identify invasion which may only be focally present and thus such tumours, particularly those of large size, must be extensively sampled for accurate diagnosis (Riopel et al, 1999; Lee and Young, 2003; Seidman et al, 2003; Ronnett et al, 2004). Moreover, it can be very difficult to differentiate primary MOC from secondary mucinous carcinomas from other sites, in particular the gastrointestinal tract (Hart, 2005). Indeed it is thought that many carcinomas diagnosed as primary mucinous epithelial ovarian cancer are likely metastatic disease, and that the true frequency of mucinous carcinoma arising in the ovary is $<3 \%$ of all ovarian carcinomas (Gilks, 2004; Seidman et al, 2004). Hence early diagnosis and accurate classification of MOC, including the ability to identify patients who are likely to progress to invasive disease, is critical to patient prognosis and treatment (Hart, 2005).

The molecular basis of MOC, including the genetic events that initiate the development of disease and those leading to malignant progression, are largely unknown. One genetic abnormality characteristic of MOC is a high frequency of mutations in KRAS, thought to occur early in the development of MOC (Feeley and Wells, 2001). Unlike serous ovarian carcinomas, mutations in p53 are rarely observed in MOC (Shih and Kurman, 2004). In our laboratory, we have successfully applied transcript profiling of whole tissue as a screening tool to determine molecular changes underlying cancer, which has led to the identification of several potential markers for prostate, ovarian and pancreatic cancer (Henshall et al, 2003a, b; Heinzelmann-Schwarz et al, 2004; Segara et al, 2005). In the current study, we have determined the gene 
expression profiles of mucinous borderline tumours and MOC using oligonucleotide microarrays representing over $90 \%$ of the expressed human genome. By comparing the results to transcript profiles of the other histological subtypes of ovarian cancer, we aimed to determine the molecular basis of mucinous ovarian tumours and to identify potential tumour markers. Following validation of the transcript profiling results using RT-PCR analysis on ovarian cancer extracts of varying histological subtypes, we determined the protein expression of one such candidate tumour marker, galectin 4 (LGALS4), in primary ovarian tissue (normal surface epithelium, benign mucinous cysts, mucinous borderline tumours and ovarian carcinomas) using high-throughput immunohistochemistry based on tissue microarrays.

\section{MATERIALS AND METHODS}

\section{Tissue and clinicopathological data}

Tissue specimens (fresh/frozen and formalin-fixed paraffinembedded samples) collected from patients undergoing primary laparotomy at the Gynaecological Cancer Centre, Royal Hospital for Women, Sydney, and the Royal North Shore Hospital, Sydney, between 1990 and 2003 were included in this study following informed consent and approval by the appropriate hospital research ethics committee. The histological classification at diagnosis was independently confirmed by a gynaecological pathologist for all tissue specimens before inclusion in the study. Normal ovaries were obtained from patients undergoing surgery for benign gynaecological conditions or unrelated malignancies. Patients exhibiting clinical, morphological or microscopic features suggesting metastatic mucinous ovarian carcinoma rather than primary MOC, including concurrent gastrointestinal carcinomas, the presence of Pseudomyxoma peritonei/ovarii, bilateral disease, Krukenberg tumours, and advanced stage borderline tumours (Lee and Young, 2003; Seidman et al, 2003; Hart, 2005), were excluded from the study. The clinical and pathological details of the tissue cohort used in this study are shown in Table 1.

\section{Molecular profiling and data analysis}

Transcript profiling was performed as previously described (Heinzelmann-Schwarz et al, 2004) using the Eos $\mathrm{Hu03,} \mathrm{a}$ customised Affymetrix GeneChip ${ }^{\mathbb{R}}$ oligonucleotide microarray containing over 59000 probesets for the interrogation of approximately 46000 unique sequences (Eos Biotechnology/ Protein Design Labs, Fremont, CA, USA; Platzer et al, 2002) using total RNA extracted from three MOC (stage I), four mucinous borderline tumours, eight endometrioid ovarian cancers, 31 serous ovarian cancers, three serous borderline tumours, and four normal ovaries. Only those tumour samples containing $>75 \%$ of borderline or invasive cancer were used for transcript profiling. Following normalisation as described (Henshall et al, 2003a), data was logtransformed before further analysis. In addition, prior to hierarchical clustering or principal components analysis, the data were scaled to ensure that each gene exhibited the same mean and variance. Principal components analysis was used to provide a visual demonstration of the variation in gene expression of the top ranked between ovarian cancer histological subtypes using the Stats package in R (http://www.r-project.org; Smyth, 2004). Hierarchical clustering of genes and samples was performed using an euclidean distance metric with average linkage (Spotfire DecisionSite 8.0).

A penalised $t$-test (Lönnstedt and Speed, 2002; Smyth, 2004) was used to identify genes differentially regulated between MOC and other subtypes of ovarian cancer. $P$-values were adjusted for multiple testing using the Benjamini-Yekutieli method (Benjamini
Table I Clinical and pathological details of the ovarian tumour cohort used for immunohistochemistry $(n=124)^{\text {a }}$

\begin{tabular}{|c|c|}
\hline Variable & No. patients (\%) \\
\hline \multicolumn{2}{|l|}{ Histological type } \\
\hline Serous & $55(44.4)$ \\
\hline Mucinous & $39(31.4)$ \\
\hline Endometrioid & $22(17.7)$ \\
\hline Clear cell & $8(6.5)$ \\
\hline \multicolumn{2}{|l|}{ FIGO stage $^{b}$} \\
\hline 1 & $27(28.4)$ \\
\hline$\|$ & $5(5.3)$ \\
\hline III & $55(57.9)$ \\
\hline IV & $8(8.4)$ \\
\hline \multicolumn{2}{|l|}{ Grade $^{c}$} \\
\hline Borderline (mucinous only) & $29(24.8)$ \\
\hline 1 - l & $20(17.1)$ \\
\hline 2 & $35(29.9)$ \\
\hline 3 & $33(28.2)$ \\
\hline \multicolumn{2}{|l|}{ Age } \\
\hline$<60$ & $62(50.0)$ \\
\hline$\geqslant 60$ & $62(50.0)$ \\
\hline \multicolumn{2}{|l|}{ Residual disease } \\
\hline$\leqslant 1$ & $92(74.2)$ \\
\hline$>1$ & $32(25.8)$ \\
\hline \multicolumn{2}{|l|}{$\mathrm{CA} / 25^{\mathrm{d}}$} \\
\hline$\leqslant 500$ & $58(60.0)$ \\
\hline$>500$ & $39(40.0)$ \\
\hline \multicolumn{2}{|l|}{ Outcome } \\
\hline Alive & $62(53.0)$ \\
\hline Death (related to malignancy) & $48(41.0)$ \\
\hline Death (unrelated or unknown cause) & $7(6.0)$ \\
\hline
\end{tabular}

a Unless otherwise stated. ${ }^{b}$ Carcinomas only; $n=95 .{ }^{c} n=117 .{ }^{d} n=97$.

and Yekutieli, 2001). Genes with an adjusted $P$-value $<0.01$ can be interpreted as having a false discovery rate of $1 \%$. Genes were assigned to functional categories (molecular function, biochemical process, cellular localisation, chromosome) using Gene Ontology (http://vortex.cs.wayne.edu/projects.htm; Draghici et al, 2003) and GenMAPP (www.genmapp.org) analysis (Dahlquist et al, 2002).

\section{RT - PCR}

RNA $(2 \mu \mathrm{g})$ was treated with DNAse then reverse-transcribed using the Reverse Transcription System (Promega, Australia) according to the manufacturer's instructions. Semi-quantitative RT-PCR was performed by the amplification of selected gene transcripts using $2 \mu \mathrm{l}$ of the resulting cDNA in a $25 \mu \mathrm{l}$ reaction volume incorporating $200 \mu \mathrm{M}$ of dNTPs (Roche, Australia), $2.5 \mathrm{mM} \mathrm{MgCl}_{2}$, $1.5 \mathrm{U}$ of Amplitaq Gold (Promega), and $1 \mu \mathrm{M}$ of each oligonucleotide pair. Oligonucleotide primers and PCR product size for each gene were as follows: LGALS4: forward $5^{\prime}$ GCTCAACGTGGG AATGTCTGTTTAC, reverse $5^{\prime}$ TTGTAGTGCTCAGCCAGGAC TATG (260 bp); MGC32871: TGAGTCACGGACTTGCAG, TTCGCCACAAACAGTATCA (260 bp); MUCDHL: AATGTGGAA CCCAGCCACA, CACGTTTCCCCTAAAGATGCT (270 bp); CDH17: CTTCACTCCCTGTGTCTTCTTATGC, CCTGTCCAAGGCTCTGTT GTAATAC (240 bp); MEP1A: GGTTTCATCTCCCACCAAATGC, AGGTACGGCTTCCTCTAACATGG (220 bp); MUC13: GCATTTGG CTACAGTGGACTCG, CTTAGGAAAGACGCTCCCTTCTG (240 bp); FABP1: GAGCCAGGAAAACTTTGAAGCC, TGGTGATTATGTCG CCGTTGAG (300 bp); C19orf21: CCAACGCCAGATGAGAACT, 
CGTTCTTGTGACGGGTC (260 bp); MUC2: CGGTAAAACGACCC CACACAAG, CATCAAAGCCAGGAGCGTAGTTC (400 bp); TFF1: TGGAGAACAAGGTGATCTGCG, AAACAGCAGCCCTTATTTGC AC (160 bp); REGIV: TATCAGAGAAGCCAGCCGATATG, TTGCA CAGGAAGTGTTGGCG (200 bp). Amplification of GAPDH (GTCCACTGGCGTGTTCACCA, GTGGCAGTGATGGCATGGAC, $260 \mathrm{bp}$ ) was used as a control. Cycling commenced with a $12 \mathrm{~min}$ heat activation at $95^{\circ} \mathrm{C}$, followed by 24 cycles of strand denaturation at $95^{\circ} \mathrm{C}$ for $30 \mathrm{~s}$, annealing at $55-60^{\circ} \mathrm{C}$ for $30 \mathrm{~s}$, and extension at $72^{\circ} \mathrm{C}$ for $1 \mathrm{~min}$. A final extension time of $7 \mathrm{~min}$ followed the last cycle. Products were separated on a $2 \%$ agarose gel in Tris-Acetate-EDTA buffer and visualised using ethidium bromide staining.

\section{Immunohistochemistry}

Protein expression of LGALS4 was determined in a cohort of fixed tissue from 124 patients with ovarian cancer (comprising $10 \mathrm{MOC}$ (independent of the samples that were transcript profiled), 55 serous ovarian cancers, 22 endometrioid ovarian cancers, eight clear cell ovarian cancers, and 29 mucinous borderline tumours; Table 1). In addition, eight benign mucinous cysts and 14 normal ovaries, some of which contained inclusion cysts (sites of enclosed metaplastic epithelium proposed as a precursor lesion for some ovarian carcinomas; Feeley and Wells, 2001), were used for immunohistochemistry. All tissues were incorporated into tissue microarrays following pathological review, with each patient represented by two to five tissue cores.

Tissue sections $(4 \mu \mathrm{m})$ were dewaxed and rehydrated according to standard protocols, and endogenous peroxidase blocked using $3 \% \mathrm{H}_{2} \mathrm{O}_{2}$. Sections were treated with proteinase $\mathrm{K}$ to facilitate antigen retrieval, followed by incubation for $1 \mathrm{~h}$ with goat antigalectin 4, 1:100 (sc19286, Santa Cruz Biotechnology, CA, USA). Bound antibody was detected using the LSAB + Kit/HRP and $\mathrm{DAB}+($ diaminobenzidine) (DAKO Cytomation) and hematoxylin counterstaining. A negative control omitted the primary antibody, and a positive (small bowel) and negative (testis, skeletal muscle) control tissue was included. Scoring was independently assessed by two observers trained in gynaecological pathology and discrepancies resolved by consensus. All cells within each core were counted and the percentage of cells staining for each core determined. The average percentage staining of multiple cores was calculated for each patient. Box and whisker plots showing staining distributions (median and 25th -75 th percentile range) were produced using the Base library in $\mathrm{R}$ (http://www.r-project.org). The median is marked as a horizontal line between the box edges, which represent the 25 th and 75th percentile values. The length of the whiskers is 1.5 times the interquartile range and values outside this range are marked as circles. Differences in protein expression were determined using the Mann-Whitney $U$-test, and correlations between gene expression and clinicopathological parameters were analysed using Fisher's exact test. A $P$-value of $\leqslant 0.05$ was required for significance. All statistical analyses were performed using Statview 4.5 software (Abacus Systems, Berkeley, CA, USA).

\section{RESULTS}

\section{MOC exhibit a gene expression profile distinct from other ovarian cancers}

Principal components analysis on the top 500 most variable genes identified by transcript profiling showed that MOC can be clearly distinguished from the other subtypes of ovarian cancer by their expression profile, and cluster more closely to endometrioid ovarian cancer than to serous carcinomas (Figure 1A), as previously observed (Schwartz et al, 2002; Hart, 2005). Using a penalised $t$-statistic, we identified 167 probesets with higher expression in MOC compared to serous and endometrioid ovarian cancers $(P$-value adjusted for multiple testing $<0.01)$ (Table 2 and Supplementary Data), and 18 probesets whose expression was lower in MOC compared to the other cancers (Supplementary Data). Hierarchical clustering illustrated that these genes can clearly separate MOC from the other subtypes of ovarian cancer, and shows that in most cases mucinous borderline tumours cluster closely with MOC (Figure 1B). Genes identified as having low expression in MOC compared to the other subtypes had similar expression levels in normal (whole) ovaries (Figure 1B), and their identification here may reflect their high expression in serous/ endometrioid ovarian cancers rather than reduced expression in MOC.

In all, 40 genes with higher expression in MOC compared to normal ovaries were identified (Table 3 ). As the normal ovaries were not microdissected before RNA extraction and profiling and therefore contain a high proportion of stromal tissue compared to epithelial cells, these genes likely reflect epithelial-specific genes expressed in MOC. Nonetheless, the majority of these genes are common to all subtypes of ovarian cancer, and several have been previously implicated in its pathogenesis, including TACSTD1 (Ep-CAM), CDH1 (E-cadherin), KLF5 (Kruppel-like factor 5) and ERB-B3 (Darai et al, 1997; Balzar et al, 1999; Maihle et al, 2002; Heinzelmann-Schwarz et al, 2004). Combining the two analyses, we identified that 13 of these 40 genes overlap with those that are upregulated in MOC compared to the other subtypes of ovarian cancer (highlighted in Table 3). Only four genes were identified as down-regulated in MOC compared to normal ovaries (adjusted $P<0.01$ ) (Table 3 ), all of which are also reduced in the other subtypes of ovarian cancer.

We next clustered the upregulated genes in MOC compared to the other subtypes by their chromosomal location and identified several genomic regions that appeared to be over-represented in MOC, including 3p21.3 (VILL, MST1R, SLC26A6, GLYCTK, FLJ20209), 7q22 (MUC3B, ACHE, MUC17, CLDN15, LOC55971), $11 \mathrm{p} 15$ (USH1C, MUCDHL, MUC2, SLC22A18), 11q13 (STATD10, PLCB3, MOGAT2), 11q24 (CTXL, KIAA1201, LOC120224, RICS), 15q14-15 (PPP1R14D, ITPKA, CKMT1, NMES1), 19p13.3 (FUT3, FLEKHJ1, C19orf21, LOC284422, GNA11), 19q13.1-13.4 (CYP2S1, FXYD3, LGALS4, CEACAM5, CEACAM6, FLJ20200. PTPRH), and 20q13 (HNF4A, BCAS1, PTK6). Chromosomes 11q24, 19q13.2, and $20 \mathrm{q} 13$ have been previously associated with a high frequency of loss of heterozygosity in MOC (Feltmate et al, 2005). Together these data suggest chromosomal amplification affecting these genomic loci in MOC. Moreover, both 3p21.3 (MST1R) and 20q13 (PTK6) contain putative oncogenes (Barker et al, 1997; Hess et al, 2003; Maggiora et al, 2003; Wang et al, 2003), which are frequently located in regions of genomic amplification in cancer.

Using RT-PCR, we determined the expression patterns of 11 selected genes in RNA extracts from whole normal ovaries, mucinous borderline tumours and ovarian cancers (Figure 2). All of the genes were confirmed as being upregulated in MOC compared to serous ovarian cancer and/or normal ovaries.

\section{MOC express genes associated with mucin production and intestinal-type epithelium}

Using Gene Ontology classifiers, we grouped the genes with upregulated expression in MOC compared to the other subtypes to identify biological processes that may specifically underlie the development and progression of MOC. Consistent with its morphological phenotype, we identified genes encoding several mucins including MUC2, MUC3A (MUC3) and MUC17 but not MUC16 (CA125). This mucin profile is similar to that of mucinous colon carcinomas, in particular the presence of $M U C 2$ and absence of MUC5A (Byrd and Bresalier, 2004; Hart, 2005). Several mucinrelated molecules involved in carbohydrate metabolism and protein glycosylation were identified including FUT3, GCNT3, SI, 

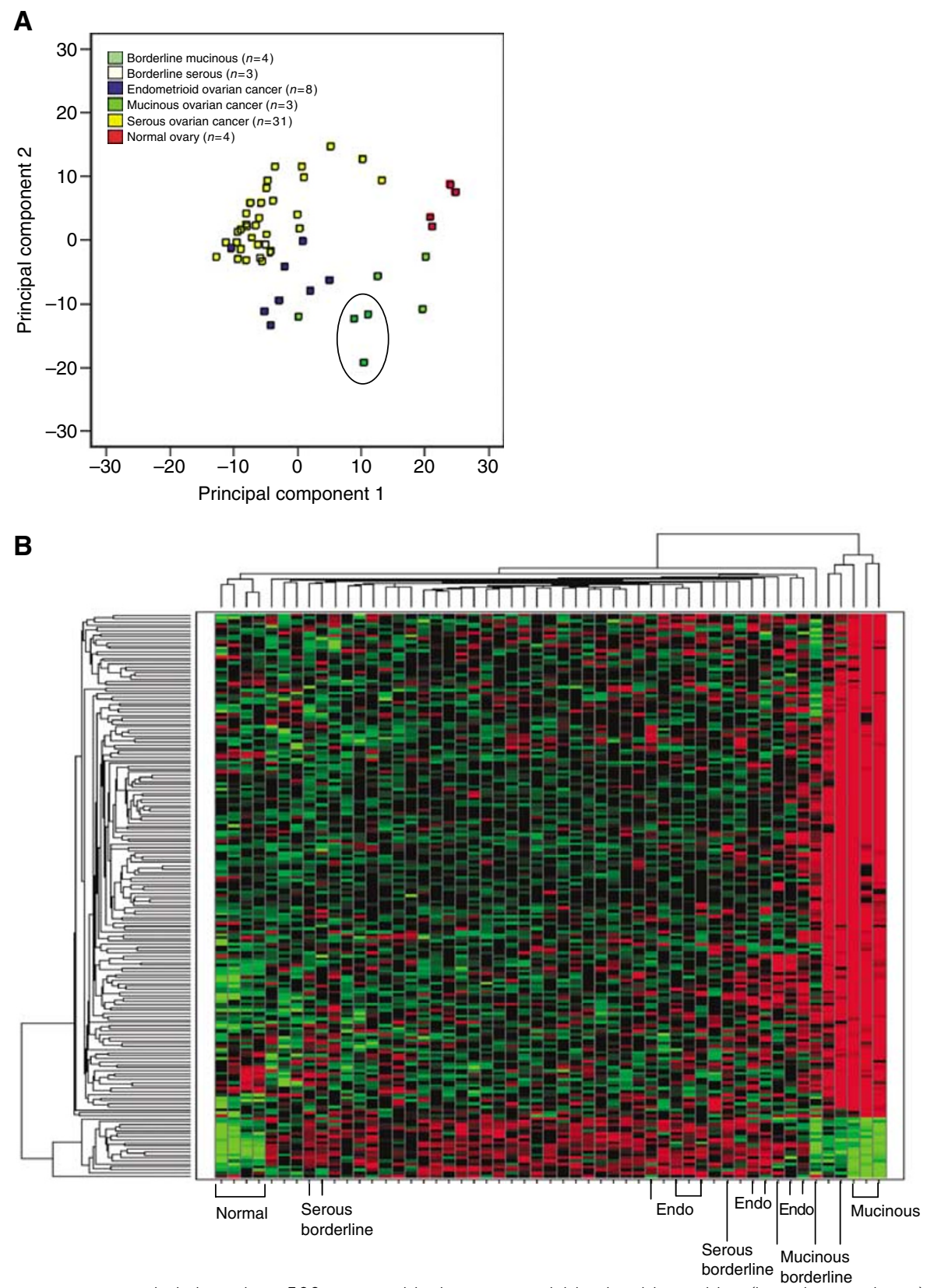

Figure I (A) Principal components analysis based on 500 genes with the most variable signal intensities (based on variance) separates the histological subtypes of EOC. MOC $(n=3)$ are circled; (B) Hierarchical clustering and heat map of differentially expressed genes $(n=167$ upregulated and $n=18$ down-regulated) in MOC compared to serous and endometrioid ovarian cancers. Clustering was performed on all transcript profiled samples ( $n=3 \mathrm{MOC}$ : $n=4$ mucinous borderline tumours; $n=8$ endometrioid ovarian cancers (endo); $n=3$ serous borderline tumours; $n=3$ I serous ovarian cancers (unlabelled columns); and four normal ovaries) as described in the Materials and Methods. Expression levels are colour coded with red, green and black corresponding to an increase, a decrease, and no change in gene expression, respectively.

FBP1, UGT1A9; and TFF1, an estrogen-regulated member of the trefoil factor family of secreted peptides associated with mucin production and frequently overexpressed in other mucinous adenocarcinomas (Emami et al, 2004).

We also identified a number of genes associated with intestinal expression including the caudal type homeobox transcription factors $C D X 1$ and $C D X 2$. CDX transcription factors are essential in intestinal epithelial development, and are also associated with oncogenesis via the modulation of various cellular processes including proliferation, apoptosis, and cell adhesion (Guo et al, 2004). Moreover, two $C D X 2$ intestinal-specific targets were identified: sucrase isomaltase (SI), a critical gene in intestinal development (Guo et al, 2004) and $C D H 17$, an enterocyte-specific cell adhesion molecule (Hinoi et al, 2002). Other intestinal-type cell adhesion molecules included LGALS4, a member of the galectin family of carbohydrate-binding molecules (Huflejt and Leffler, 2004); three members of the transmembrane 4 (tetraspanin) superfamily (TM4SF4/IL-TMP, TM4SF5/L6H and TM4SF3/ CO-029) associated with cellular proliferation, adhesion, motility, and tumour cell metastasis (Wright et al, 2000); and two members of the carcinoembryonic antigen family, CEACAM6 and CEACAM5 (CEA), frequently expressed by at least a subset of MOC (McCluggage, 2000). The identification of intestinal-type adhesion factors suggests that altered cell adhesion is a feature of MOC, 
Table 2 Genes ( $n=50$ of 167 probesets $^{\mathrm{a}}$ ) identified as upregulated in MOC compared to other histological subtypes of ovarian cancer (ranked by adjusted $P<0.01$ )

\begin{tabular}{|c|c|c|c|c|c|}
\hline Rank & Symbol $^{b}$ & Name & Unigene $^{c}$ & Locus link & Location \\
\hline । & LGALS4 & Lectin, galactoside-binding, soluble, 4 (galectin 4) & Hs.5302 & 3960 & $|9 q| 3.2$ \\
\hline 2 & & cDNA clone IMAGE:5759948, partial cds & Hs.447537 & NA & $15 q|5|$. \\
\hline 3 & & Hypothetical protein MGC3287| & Hs.242014 & | 19467 & $10 q 26.3$ \\
\hline 4 & MUCDHL & Mucin and cadherin-like & Hs. 165619 & 53841 & ||$p \mid 5.5$ \\
\hline 5 & & apobec-I complementation factor & Hs.8349 & 29974 & I0q21.1 \\
\hline 6 & $\mathrm{CDH} / 7$ & Cadherin 17, LI cadherin (liver-intestine) & Hs.89436 & 1015 & $8 q 22.1$ \\
\hline 7 & MEPIA & Meprin A, alpha (PABA peptide hydrolase) & Hs. 179704 & 4224 & $6 p|2-p| 1$ \\
\hline 8 & MUCI3 & Mucin 13, epithelial transmembrane & Hs.5940 & 56667 & $3 q 21$ \\
\hline 9 & FABPI & Fatty acid binding protein I, liver & Hs.380I35 & 2168 & $2 p \mid 1$ \\
\hline 10 & MUC3B & Mucin 3B, intestinal & Hs.489354 & NA & $7 q 22$ \\
\hline | | & CEACAM5 & CEA-related cell adhesion molecule 5 (CEA) & Hs.220529 & 1048 & $19 q \mid 3.2$ \\
\hline 12 & PDZK2 & PDZ domain containing 2 & Hs.374726 & 79849 & | | q23.3 \\
\hline 13 & GPA33 & Glycoprotein A33 (transmembrane) & Hs.437229 & 10223 & |q24. 1 \\
\hline 14 & RNFI28 & Ring finger protein 128 & Hs.496542 & 79589 & Xq22.3 \\
\hline 15 & MUCDHL & Mucin and cadherin-like & Hs. 165619 & 53841 & ||$p \mid 5.5$ \\
\hline 16 & EPS8L3 & EPS8-like 3 & Hs.485352 & 79574 & Ip|3.2 \\
\hline 17 & BCL2LI4 & BCL2-like 14 (apoptosis facilitator) & Hs.504794 & 79370 & $12 p \mid 3.2$ \\
\hline 18 & SYTL2 & Synaptotagmin-like 2 , transcript variant a & Hs.369520 & 54843 & ||$q|4|$. \\
\hline 19 & HMGCS2 & 3-hydroxy-3-methylglutaryl-Coenzyme A synthase 2 (mitochondrial) & Hs.59889 & 3158 & $|p| 3-p \mid 2$ \\
\hline 20 & FAM3D & Family with sequence similarity 3 , member $D$ & Hs.61265 & $131 \mid 77$ & $3 p 21.2$ \\
\hline 21 & MYOIA & Myosin IA & Hs.5394 & 4640 & $12 q 13.3$ \\
\hline 22 & SLC26A3 & Solute carrier family 26, member 3 & Hs. 1650 & $181 \mid$ & $7 q 31$ \\
\hline 23 & ATPIOB & ATPase, Class V, type IOB & Hs. 109358 & 23120 & $5 q 34$ \\
\hline 24 & BTNL8 & Butyrophilin-like 8 & Hs. 189109 & 79908 & $5 q 35.3$ \\
\hline 25 & MYO7B & Myosin VIIB & Hs. 154578 & 4648 & $2 q \mid 4.3$ \\
\hline 26 & CDXI & Caudal type homeo box transcription factor I & Hs. 1545 & 1044 & $5 q 33.1$ \\
\hline 27 & & cDNA clone IMAGE:466/388, partial cds & Hs.30672I & 400573 & $17 p|3|$. \\
\hline 28 & & ESTs; moderate similarity to protein P39I88 (H.sapiens) & Hs.282795 & NA & $|0 q| 1.23$ \\
\hline 29 & & ESTs & Hs. I 6462 & NA & $20 q 13.12$ \\
\hline 30 & & Cisplatin resistance associated & Hs.425I 44 & 10903 & $1 \mathrm{q} 21.2$ \\
\hline 31 & TRIM3। & Tripartite motif-containing 31 & Hs.493275 & 11074 & $6 p 21.3$ \\
\hline 32 & UGTIA9 & UDP glycosyltransferase I family, polypeptide A9 & Hs. 124112 & 54600 & $2 q 37$ \\
\hline 33 & $\mathrm{ACHE}$ & Acetylcholinesterase (YT blood group) & NA & 43 & $7 q 22$ \\
\hline 34 & PLA2GIO & Phospholipase $A 2$, group $X$ & Hs. 144442 & 401831 & $|6 p| 3.1-p \mid 2$ \\
\hline 35 & & Hypothetical protein LOCI44347 & Hs.43290I & 144347 & $12 q 24.31$ \\
\hline 36 & GUCY2C & Guanylate cyclase $2 \mathrm{C}$ (heat stable enterotoxin receptor) & Hs.524278 & 2984 & $12 p \mid 2$ \\
\hline 37 & & cDNA clone IMAGE:4806358, partial cds & Hs.328236 & NA & $4 q 32.3$ \\
\hline 38 & PLAC8 & Placenta-specific 8 & Hs.371003 & 51316 & $4 q 21.3$ \\
\hline 39 & & KIAA0828 protein & Hs. 195058 & 23382 & $7 q 32.3$ \\
\hline 40 & COLI7AI & Collagen, type XVII, alpha I & Hs. I 17938 & 1308 & I0q25.1 \\
\hline 41 & CLCAI & Chloride channel, calcium activated, family member I & Hs. 194659 & 1179 & $|p 3|-p 22$ \\
\hline 42 & & Hypothetical protein FLJ20225 & Hs. I24835 & 54546 & $1 p 36.13$ \\
\hline 43 & TM4SF4 & Transmembrane 4 superfamily member 4 & Hs. 133527 & 7104 & $3 q 25$ \\
\hline 44 & MUCI7 & mucin 17 & Hs.271819 & 140453 & $7 q 22$ \\
\hline 45 & $\mathrm{FA} 2 \mathrm{H}$ & Fatty acid 2-hydroxylase & $H s .461329$ & 79152 & $16 q 23$ \\
\hline 46 & HNF4A & Hepatocyte nuclear factor 4 , alpha & Hs. I 6462 & 3172 & $20 q|2-q| 3.1$ \\
\hline 47 & SEMA4G & $\begin{array}{l}\text { Sema domain, immunoglobulin domain, transmembrane domain and short } \\
\text { cytoplasmic domain, (semaphorin) } 4 G\end{array}$ & Hs.444359 & 57715 & $10 q 24.32$ \\
\hline 48 & & cDNA FLJ26898 fis, clone RCT00475 & Hs.19937I & NA & ||$q|3|$. \\
\hline 49 & C9orfl52 & Chromosome 9 open reading frame 152 & Hs. 125608 & 401546 & $9 q 32$ \\
\hline 50 & SIOOP & SI00 calcium binding protein $\mathrm{P}$ & Hs.2962 & 6286 & $4 p 16$ \\
\hline
\end{tabular}

${ }^{a}$ Full list of genes are listed in Supplementary Data. ${ }^{b} \mathrm{HUGO}$. 'Unigene identifiers were derived from the UniGene Build \#176 (October 2004).

similar to other histological subtypes of ovarian cancer (Heinzelmann-Schwarz et al, 2004). Moreover, several of these adhesion factors have been previously implicated in carcinogenesis, including TM4SF and CEACAM family members (Scholzel et al, 2000; Wright et al, 2000; Ilantzis et al, 2002), CDH17 (Grotzinger et al, 2001; Takamura et al, 2004), and LGALS4 (Huflejt and Leffler, 2004).

\section{Cellular pathways underlying MOC development}

Gene Ontology analysis identified a number of genes involved in cellular processes associated with cancer, including cell adhesion, signalling, proliferation, and apoptosis (Table 4). Several putative oncogenes were differentially expressed in MOC, including the breast tumour kinase BRK (PTK6) (Barker et al, 1997) not previously implicated in ovarian cancer pathogenesis; and MST1R/ $R O N$, a receptor tyrosine kinase associated with proliferation and motility of cancer cells including ovarian carcinoma (Hess et al, 2003; Maggiora et al, 2003; Wang et al, 2003).

Although KRAS mutations are associated with MOC (Shih and Kurman, 2004), we did not find any evidence of increased KRAS activity at the transcriptional level. Using GenMAPP analysis, we examined if any probesets corresponding to other members of the mitogen activated protein (MAP) kinase cascade were differentially expressed in MOC compared to the other subtypes of ovarian cancer. This revealed a slight increase in ERK1 (1.19-fold change, 
Table 3 Genes identified as $(A)$ up-regulated $(n=40)$ and $(B)$ down-regulated $(n=4)$ in MOC compared to normal ovaries (ranked by adjusted $P<0.0 \mathrm{I})$. Genes highlighted in bold $(n=13)$ are also up-regulated in MOC compared to other subtypes of ovarian cancer

\begin{tabular}{|c|c|c|c|c|c|}
\hline Rank & Symbol & Name & Unigene & Locus Link & Location \\
\hline \multicolumn{6}{|c|}{ (A) Upregulated } \\
\hline I & LGALS4 & Lectin, galactoside-binding, soluble, 4 (galectin 4 ) & Hs.5302 & 3960 & $19 q 13.2$ \\
\hline 2 & & Hypothetical protein FLJ20171 & Hs.48747I & 54845 & $8 q 22.1$ \\
\hline 3 & TACSTDI & Tumor-associated calcium signal transducer I (Ep-CAM) & Hs.692 & 4072 & $2 p 21$ \\
\hline 4 & ERBB3 & v-erb-b2 erythroblastic leukemia viral oncogene homolog 3 & Hs.30625I & 2065 & $12 q 13$ \\
\hline 5 & & cDNA clone IMAGE:5759948, partial cds & Hs.447537 & NA & $|5 q| 5 . \mid$ \\
\hline 6 & BCMPII & Breast cancer membrane protein II & Hs. 100686 & 155465 & $7 p 21.1$ \\
\hline 7 & $\mathrm{CXXC5}$ & CXXC finger 5 & Hs. 189119 & 51523 & $5 q 31.2$ \\
\hline 8 & $\mathrm{EHF}$ & Ets homologous factor & Hs.502306 & 26298 & ||$p \mid 2$ \\
\hline 9 & STARDIO & START domain containing 10 & Hs. I 88606 & 10809 & ||$q \mid 3$ \\
\hline 10 & CI9orf2I & Chromosome 19 open reading frame 21 & Hs.439180 & 126353 & $19 p \mid 3.3$ \\
\hline|| & MUCI3 & Mucin 13, epithelial transmembrane & Hs.5940 & 56667 & $3 q 21.2$ \\
\hline 12 & PROMI & Prominin 1 & Hs.479220 & 8842 & $4 p \mid 5.32$ \\
\hline 13 & MUCDHL & Mucin and cadherin-like & Hs. 165619 & 53841 & ||$p \mid 5.5$ \\
\hline 14 & & Hypothetical gene supported by BC022385; BC035868; BC048326 & Hs.390599 & 440335 & $16 p 13.3$ \\
\hline 15 & CEACAM5 & Carcinoembryonic antigen-related cell adhesion molecule 5 (CEA) & Hs.220529 & 1048 & $19 q|3.1-q| 3.2$ \\
\hline 16 & AGPAT2 & I-acylglycerol-3-phosphate O-acyltransferase 2 & Hs.320I5I & 10555 & $9 q 34.3$ \\
\hline 17 & & Hypothetical protein MGC3287| & Hs.2420l4 & | 19467 & 10q26.2 \\
\hline 18 & & Hypothetical protein MGCI 1242 & Hs.368260 & 79170 & $17 q 21.32$ \\
\hline 19 & CLDN7 & Claudin 7 & Hs.513915 & 1366 & $17 p \mid 3$ \\
\hline 20 & & FLJ46072 protein & Hs.67776 & 286077 & $8 q 24.3$ \\
\hline 21 & CALML4 & Calmodulin-like 4; breast cancer antigen NY-BR-20 & Hs.435457 & 91860 & $15 q 23$ \\
\hline 22 & PLSI & Plastin I (I isoform) & Hs.203637 & 5357 & $3 q 23$ \\
\hline 23 & KLF5 & Kruppel-like factor 5 (intestinal) & Hs.508234 & 688 & | 3q22.1 \\
\hline 24 & KIAAOIOI & KIAAOIOI & Hs.81892 & 9768 & $15 q 22.31$ \\
\hline 25 & TSPAN-I & Tetraspan I & Hs.38972 & 10103 & |p34.| \\
\hline 26 & TDE2L & Tumor differentially expressed 2-like & Hs.270655 & 347735 & |p35. | \\
\hline 27 & AGR2 & Anterior gradient 2 homolog (Xenopus laevis) & Hs.530009 & $|055|$ & $7 p 21.3$ \\
\hline 28 & ARHGAP27 & Rho GTPase activating protein 27 & Hs.463165 & 201176 & |7q21.31 \\
\hline 29 & NMESI & Normal mucosa of esophagus specific I & Hs. I 12242 & 84419 & $15 q 21.1$ \\
\hline 30 & CEACAMI & Carcinoembryonic antigen-related cell adhesion molecule I & Hs.5I2682 & 634 & $19 q 13.2$ \\
\hline 31 & $\mathrm{STI} 4$ & Suppression of tumorigenicity I4 (colon carcinoma, matriptase, epithin) & Hs.5043I 5 & 6768 & | | q24-q25 \\
\hline 32 & & LOC387882 hypothetical protein & Hs. 525657 & 387882 & $12 q 23.3$ \\
\hline 33 & TRPM4 & Transient receptor potential cation channel, subfamily M, member 4 & Hs.467I0I & 54795 & $19 q 13.33$ \\
\hline 34 & BCLP & Beta-casein-like protein & Hs.53452I & | 13452 & Ip35-p34 \\
\hline 35 & $\mathrm{IFI} 30$ & Interferon, gamma-inducible protein 30 & Hs. 14623 & 10437 & $19 p|3|$. \\
\hline 36 & $\mathrm{ABPI}$ & Amiloride binding protein I (amine oxidase (copper-containing)) & Hs.521296 & 26 & $7 q 34-q 36$ \\
\hline 37 & ERBB3 & v-erb-b2 erythroblastic leukemia viral oncogene homolog 3 & Hs.30625I & 2065 & $12 q 13$ \\
\hline 38 & MAL2 & Mal, T-cell differentiation protein 2 & Hs.20l083 & | 14569 & $8 q 24.12$ \\
\hline 39 & CDHI7 & Cadherin I7, LI cadherin (liver-intestine) & Hs.89436 & 1015 & $8 q 22.1$ \\
\hline 40 & $\mathrm{CDHI}$ & Cadherin I, type I, E-cadherin (epithelial) & Hs.46I086 & 999 & 16q22.1 \\
\hline \multicolumn{6}{|c|}{ (B) Downregulated } \\
\hline i & & Similar to lymphocyte antigen 6 complex, locus G5B; G5b protein & Hs.23650 & | 12476 & $|6 p| 1.2$ \\
\hline 2 & RARRES2 & Retinoic acid receptor responder (tazarotene induced) 2 (TIG2) & Hs.52I 286 & 5919 & $7 q 36.1$ \\
\hline 3 & PDGFD & Platelet derived growth factor D & Hs.352298 & 80310 & | | q22.3 \\
\hline 4 & & Hypothetical protein MGCII36 & Hs.8719 & 78986 & $8 p 12$ \\
\hline
\end{tabular}

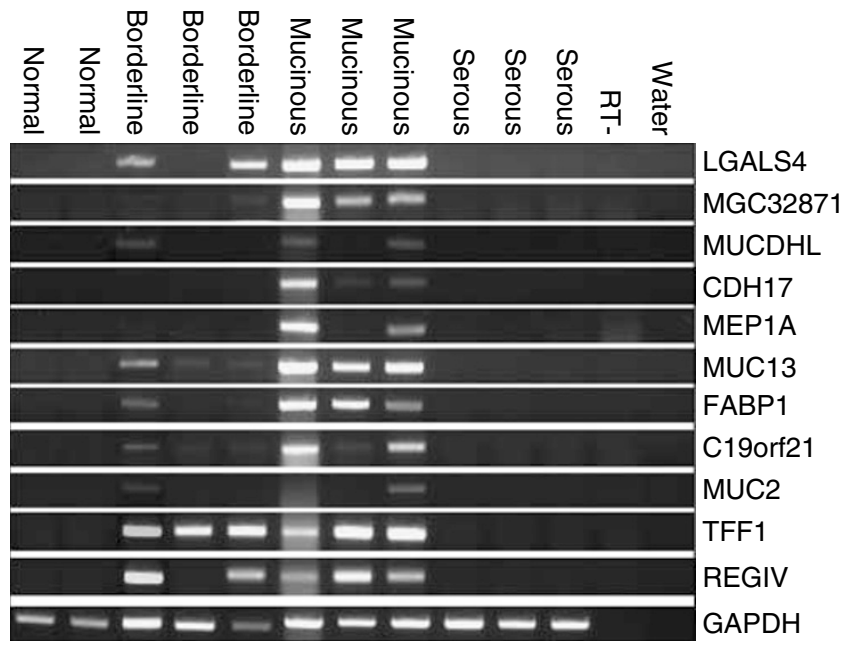

unadjusted $P<0.001)$ and a two-fold decrease in MAP kinase kinase 1 (MEKK1) expression (0.61-fold change, unadjusted $P=0.03)$, the latter being linked to cisplatin-resistance in ovarian cancer (Gebauer et al, 2000), a feature of MOC (Hess et al, 2004).

\section{LGALS4 is specifically expressed in MOC}

LGALS4 is an intestinal cell surface adhesion molecule that is overexpressed in intestinal carcinomas (Grotzinger et al, 2001). The results of the transcript profiling experiment suggested that LGALS4 was also highly overexpressed in MOC (Table 2,

Figure 2 Semi-quantitative RT-PCR analysis of RNA expression in normal ovaries $(n=2)$, mucinous borderline tumours $(n=3)$, mucinous ovarian cancers $(n=3)$ and serous ovarian cancers $(n=3)$. RT-, no reverse transcriptase control; water, no cDNA. For gene descriptions, see Table 2 and Supplementary Data. 
Table 4 Selected categories/genes from Gene Ontology (GO) analysis of genes specifically upregulated in MOC compared to other histological subtypes of ovarian cancer

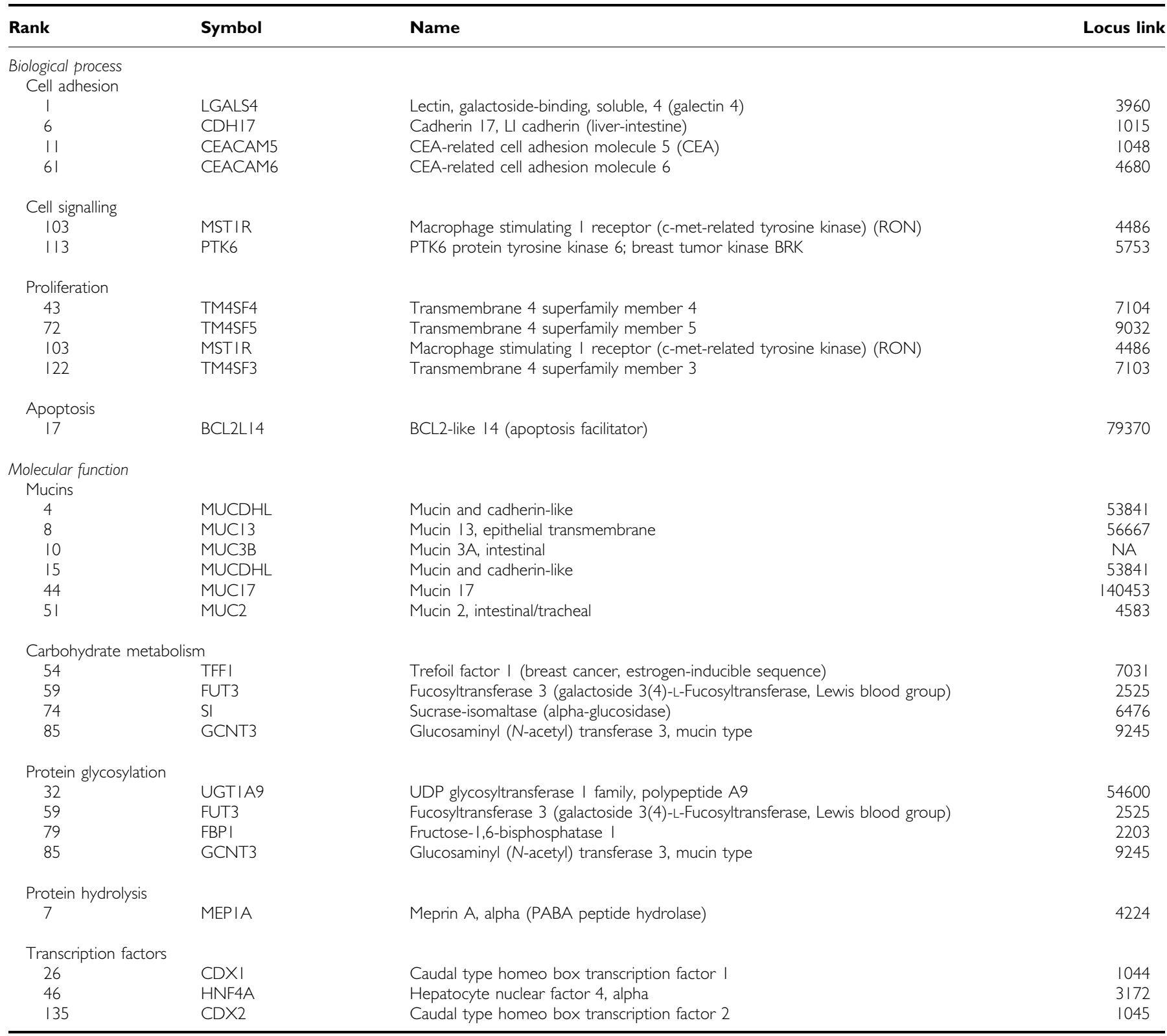

Figure 3A). Moreover, LGALS4 is located at $19 \mathrm{q} 13.3$, a region associated with a high frequency of loss of heterozygosity in MOC (Feltmate et $a l, 2005$ ) and where we identified a cluster of upregulated genes. We therefore examined the expression of LGALS4 in ovarian carcinoma using immunohistochemistry (Table 1, Figure 3B). In accordance with the transcript profiling results, expression of LGALS4 was highly and specifically expressed in MOC (median expression $72 \%$ of cells staining positive) compared to the other ovarian carcinoma subtypes (serous and endometroid, $P<0.001$; clear cell $P=0.002$ ) and to normal ovarian surface epithelium $(P=0.002)$, all of which had a median expression equivalent to zero (Figure $4 \mathrm{~A}$ ). To identify if LGALS4 expression occurs early in disease onset, we examined its expression in benign mucinous cysts and mucinous borderline tumours, in addition to low- and high-stage MOC. LGALS4 expression was detected at a median expression level of approximately $30 \%$ of cells staining in benign mucinous cysts, increasing in borderline tumours to similar levels of expression as in MOC ( $>70 \%$ of cells; Figure $4 \mathrm{~B}$ ). There was no significant difference in expression between borderline tumours and low grade MOC $(P=0.47)$, and although a decrease in expression from low- to high-stage MOC was observed, this was not statistically significant $(P=0.21)$. Statistical analysis did not reveal any correlation between LGALS4 expression and clinicopathological parameters (age, grade, stage, outcome; Table 1) in the ovarian mucinous tumour cohort (data not shown).

\section{DISCUSSION}

Mucinous ovarian cancers are one of the less common histological subtypes of ovarian carcinoma. Combined with the difficulty in accurate diagnosis of primary disease, its relative rarity has contributed to the lack of knowledge regarding the molecular basis 
A

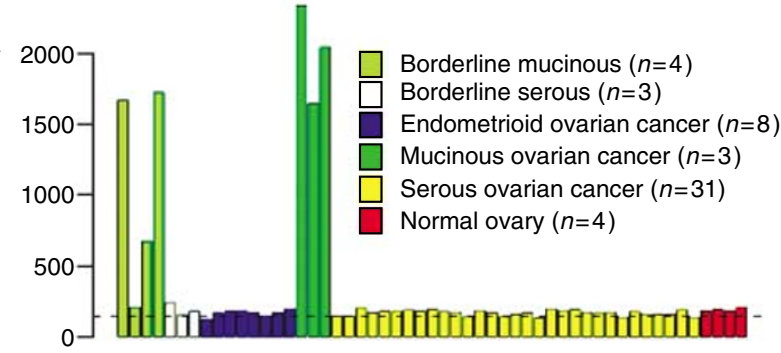

B

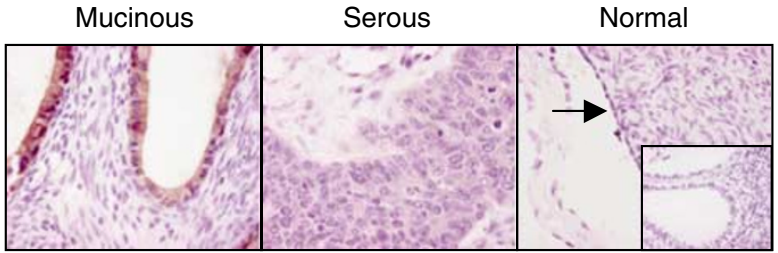

Figure 3 (A) mRNA transcript profile for LGALS4. The dashed line represents the signal intensity of the 15 th percentile of the gene expression in normal body tissues (Henshall et al, 2003a); (B) representative immunohistochemistry staining for LGALS4 in MOC, serous ovarian cancer, normal ovarian surface epithelium (arrowed) and epithelial inclusion cysts (inset); $\times 40$ magnification.
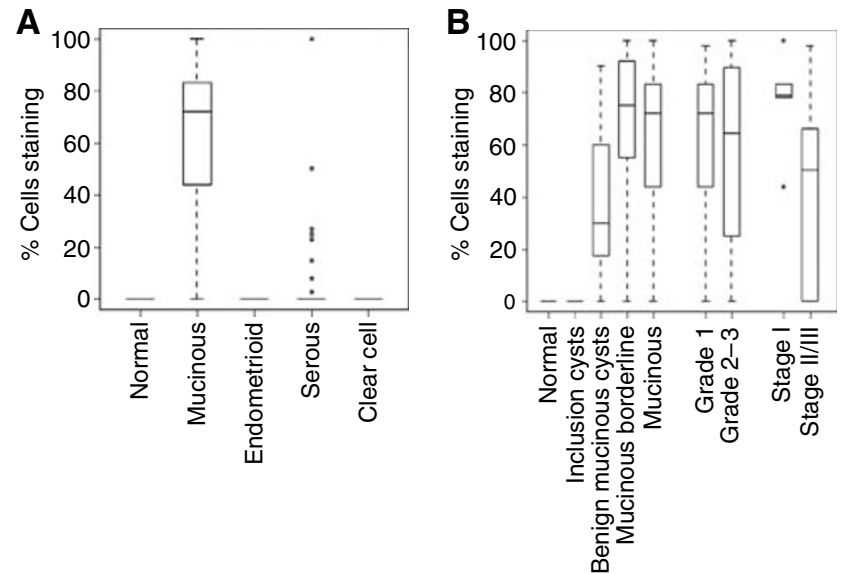

Figure 4 Box plots showing distribution of expression of LGALS4 in (A) normal ovarian surface epithelium $(n=14)$ and in different histological subtypes of ovarian carcinoma: MOC $(n=10)$; endometrioid ovarian cancer $(n=22)$; serous ovarian cancer $(n=55)$; clear cell ovarian cancer $(n=8)$; and $(\mathbf{B})$ in epithelial ovarian inclusion cysts $(n=8)$; benign mucinous cysts $(n=8)$; mucinous borderline tumours $(n=29)$; low- (grade $1 ; n=6$ ) and high-grade (grade $2-3 ; n=4$ ) MOC; and low- (stage $1 ; n=5$ ) and high-stage MOC (stage $\|-\| I \mid ;=5$ ). For explanation of box plots, see Materials and Methods.

of its development and progression. In this study, we have shown that MOC show a gene expression profile that both overlaps with and is distinct from the other histological subtypes of ovarian carcinoma, presumably reflecting their common ovarian origin but different morphological and clinical presentations. In particular, we found that MOC express genes that underlie their morphological phenotype, including intestinal-specific genes, which likely reflects the intestinal-type differentiation characteristic of most MOC (Feeley and Wells, 2001). A variety of mucin molecules are also expressed in MOC. Alterations in expression of mucins, including loss of organ specificity, are a common feature of cancer and are associated with altered biological properties including metastatic potential (Byrd and Bresalier, 2004). In addition, variations in mucin glycosylation patterns can cause changes in tumour cell adhesion, migration and invasion (Casey et al, 2003), and can be mediated by the differential expression of glycosylation enzymes including fucosyltransferases and sialyltransferases. We identified several such enzymes, suggesting specific mucin glycosylation patterns are a feature of MOC.

It is likely that many carcinomas diagnosed as primary mucinous ovarian cancer are more likely to be metastatic disease originating in the gastrointestinal tract (Gilks, 2004; Seidman et al, 2004). In this study, we were particularly careful to only include patients that clearly fit with current clinical and histological guidelines as primary MOC rather than metastatic disease (Seidman et al, 2003; Hart, 2005). However, these strict selection criteria, combined with the relative rarity of MOC, resulted in a small sample number both for the transcript profiling and validation experiments. In addition, although comprising at least $75 \%$ tumour cells, the tissue samples used in the transcript profiling experiments were not microdissected and therefore may contain a small proportion of stromal elements. Therefore, our results remain to be validated in independent studies. To this end, several published studies have reported similar findings in regard to the genetic profile of MOC. First, Schwartz et al (2002) used principal components analysis to show that gene expression profiles could distinguish MOC from serous ovarian cancer, with some overlap with endometrioid ovarian cancer. Secondly, using cDNA arrays incorporating 9121 elements, Ono et al (2000) identified 115 genes that were differentially regulated between serous ovarian carcinomas and MOC). By comparing the Unigene/ Locus Link identifiers corresponding to the GenBank accessions in the Ono study to the gene identifiers in our study (Table 2 and Supplementary Data), we identified only one gene (TUBB2; tubulin beta 2) that overlaps between our lists of differentially expressed genes. A more recent study reported 46 genes that were overexpressed in MOC compared to the other histological subtypes of ovarian carcinoma and to normal ovarian surface epithelium (Marquez et al, 2005). Fifteen of those genes (TM4SF3, S100P, TM4SF4, CEACAM6, LGALS4, CEACAM5, TUBB, CTSE, GCNT3, REG4, FABP1, SDCBP2, TFF1, RNF128, PLAC8) were also identified in our study. Moreover, we also showed that LGALS4 is consistently highly expressed in MOC but is absent in the other histological subtypes of ovarian cancer and normal ovaries using immunohistochemistry, thus confirming the transcript profiling results.

Progression from borderline tumours and low-stage carcinoma to advanced MOC is associated with a poor outcome; hence the identification of tumour markers that can detect early disease, together with those that can predict patients likely to progress to advanced stage MOC, would have a major impact on patient prognosis. We determined that LGALS4 is not expressed in normal ovarian surface epithelium but is expressed at high levels in mucinous borderline tumours and in benign mucinous cysts, consistent with activation of expression early in MOC development. We did not identify any genes including LGALS4 that were significantly differentially expressed between mucinous borderline tumours and MOC, suggesting that there may be very few or only subtle changes in gene expression between mucinous borderline tumours and low-stage MOC (which were used in the transcript profiling analysis), concordant with their similar outcomes. A study with sufficient power to compare high-stage MOC to borderline and low-stage MOC may reveal gene expression changes that correlate with the poor prognosis in these patients.

There are currently no specific or sensitive serum markers for the diagnosis of MOC (Rapkiewicz et al, 2004). MOC often fail to express the ovarian cancer serum marker CA125 (MUC16), which is frequently elevated in the serum of patients with nonmucinous ovarian carcinoma. Although a cell surface adhesion molecule, LGALS4 has at least a partial extracellular component (Huflejt and Leffler, 2004), but to our knowledge there is no report of its 
presence in serum. Serum antibodies against LGALS4 have, however, been reported in a patient with colorectal cancer (Scanlan et al, 1998). Given the high level of LGALS4 expression in MOC, one might predict that similar antibodies could be detected in patients with MOC, which is currently under investigation. Hence LGALS4 may have application as an early serum diagnostic marker of MOC, either alone or in combination with other markers such as CEA (CEACAM5) and CA19.9 (Rapkiewicz et al, 2004; Hart, 2005). In addition, the high level of LGALS4 expression in MOC may aid in the histological differentiation of primary MOC from metastatic ovarian carcinoma arising at other sites (Heinzelmann-Schwarz et al; manuscript submitted for publication).

As previously suggested (Hess et al, 2004), the obvious genetic similarities of MOC with mucinous-type intestinal carcinomas support a move toward the use of a therapeutic approach tailored to the molecular characteristics of MOC rather than the tissue of origin. Patients with advanced stage MOC generally receive the same adjuvant chemotherapy as the other subtypes of ovarian carcinoma, normally a platinum-based approach combined with paclitaxel. The poor survival associated with advanced MOC may reflect a failure to respond to this regime (Hess et al, 2004). Hence, alternative combination chemotherapy regimes that target both the ovarian and mucinous intestinal genetic components of MOC, such as a platin compound combined with 5-fluorouracil, commonly used in the treatment of intestinal carcinomas, may prove to be more efficacious for MOC. This, however, remains to be tested in appropriate clinical trials.

\section{REFERENCES}

Balzar M, Winter MJ, de Boer CJ, Litvinov SV (1999) The biology of the 171A antigen (Ep-CAM). J Mol Med 77: 699-712

Barker KT, Jackson LE, Crompton MR (1997) BRK tyrosine kinase expression in a high proportion of human breast carcinomas. Oncogene 15: $799-805$

Benjamini Y, Yekutieli D (2001) The control of the false discovery rate in multiple hypothesis testing under dependency. Annals Statistics 29: $1165-1188$

Byrd JC, Bresalier RS (2004) Mucins and mucin binding proteins in colorectal cancer. Cancer Metastasis Rev 23: 77-99

Casey RC, Oegema Jr TR, Skubitz KM, Pambuccian SE, Grindle SM, Skubitz AP (2003) Cell membrane glycosylation mediates the adhesion, migration, and invasion of ovarian carcinoma cells. Clin Exp Metastasis 20: $143-152$

Dahlquist KD, Salomonis N, Vranizan K, Lawlor SC, Conklin BR (2002) GenMAPP, a new tool for viewing and analyzing microarray data on biological pathways. Nature Genet 31: 19-20

Darai E, Scoazec JY, Walker-Combrouze F, Mlika-Cabanne N, Feldmann G, Madelenat P, Potet F (1997) Expression of cadherins in benign, borderline, and malignant ovarian epithelial tumors: a clinicopathologic study of 60 cases. Hum Pathol 28: $922-928$

Draghici S, Khatri P, Martins RP, Ostermeier GC, Krawetz SA (2003) Global functional profiling of gene expression. Genomics 81: 98-104

Emami S, Rodrigues S, Rodrigue CM, Le Floch N, Rivat C, Attoub S, Bruyneel E, Gespach C (2004) Trefoil factor family (TFF) peptides and cancer progression. Peptides 25: 885-898

Feeley KM, Wells M (2001) Precursor lesions of ovarian epithelial malignancy. Histopathol 38: 87-95

Feltmate CM, Lee KR, Johnson M, Schorge JO, Wong K-K, Hao K, Welch WR, Bell DA, Berkowitz RS, Mok SC (2005) Whole-genome allelotyping identified distinct loss-of-heterozygosity patterns in mucinous ovarian and appendiceal carcinomas. Clin Cancer Res 11: 7651-7657

Gebauer G, Mirakhur B, Nguyen Q, Shore SK, Simpkins H, Dhanasekaran N (2000) Cisplatin-resistance involves the defective processing of MEKK1 in human ovarian adenocarcionoma 2008/C13 cells. Int J Oncol 16: $321-325$

Gilks CB (2004) Subclassification of ovarian surface epithelial tumors based on correlation of histologic and molecular pathologic data. Int J Gynecol Pathol 23: 200-205

\section{ACKNOWLEDGEMENTS}

This study is part of the Ovarian Cancer Project, a collaboration between the Royal Hospital for Women Gynaecological Cancer Centre and the Cancer Research Program, Garvan Institute of Medical Research. The authors wish to thank Professor Donald Marsden and Dr Greg Robertson, Consultant Gynaecological Oncologists at the Gynaecological Cancer Centre, Royal Hospital for Women; and Dr's Catherine Camaris and Lyndal Edwards, Department of Pathology, Royal Hospital for Women for their professional assistance in tissue harvesting and histopathology, essential to carrying out this study. We also thank Dr's Kurt Gish and David Mack, Eos Biotechnology/Protein Design Labs, Fremont CA for assistance with transcript profiling. This study was supported by the Gynaecological Oncology (GO) Fund, Royal Hospital for Women Foundation, Randwick NSW 2031 Australia. VHS was supported by a scholarship from the Swiss National Foundation (FNSNF No. 81AM-068430) and the Division of Gynecology, University Hospital Zurich. POB and SMH are Fellows of the Cancer Institute New South Wales. RLS and SMH are also supported by the National Health \& Medical Research Council of Australia, The Cancer Council New South Wales, the RT Hall Trust, and the Prostate Cancer Foundation of Australia (SMH).

Supplementary Information accompanies the paper on the British Journal of Cancer website (http://www.nature.com/bjc)

Grotzinger C, Kneifel J, Patschan D, Schnoy N, Anagnostopoulos I, Faiss S, Tauber R, Wiedenmann B, Gessner R (2001) LI-cadherin: a marker of gastric metaplasia and neoplasia. Gut 49: 73-81

Guo RJ, Suh ER, Lynch JP (2004) The role of CDX proteins in intestinal development and cancer. Cancer Biol Ther 3: 593-601

Hart WR (2005) Diagnostic challenge of secondary (metastatic) ovarian tumors simulating primary endometrioid and mucinous neoplasms. Pathol Int 55: $231-243$

Heinzelmann-Schwarz VA, Gardiner-Garden M, Henshall SM, Scurry J, Scolyer RA, Davies MJ, Heinzelmann M, Kalish LH, Bali A, Kench JG, Vanden Bergh PM, Hacker NF, Sutherland RL, O'Brien PM (2004) Overexpression of the cell adhesion molecules DDR1, claudin 3 and Ep-CAM in metaplastic ovarian epithelium and ovarian cancer. Clin Cancer Res 10: 4427 - 4436

Henshall SM, Afar DE, Hiller J, Horvath LG, Quinn DI, Rasiah KK, Gish K, Willhite D, Kench JG, Gardiner-Garden M, Stricker PD, Scher HI, Grygie JJ, Agus DB, Mack DH, Sutherland RL (2003a) Survival analysis of genome-wide gene expression profiles of prostate cancers identifies new prognostic targets of disease relapse. Cancer Res 63: 4196-4203

Henshall SM, Afar DE, Rasiah KK, Horvath LG, Gish K, Caras I, Ramakrishnan V, Wong M, Jeffry U, Kench JG, Quinn DI, Turner JJ, Delprado W, Lee CS, Golovsky D, Brenner PC, O’Neill GF, Kooner R, Stricker PD, Grygiel JJ, Mack DH, Sutherland RL (2003b) Expression of the zinc transporter ZnT4 is decreased in the progression from early prostate disease to invasive prostate cancer. Oncogene 22: $6005-6112$

Hess KA, Waltz SE, Toney-Earley K, Degen SJ (2003) The receptor tyrosine kinase Ron is expressed in the mouse ovary and regulates inducible nitric oxide synthase levels and ovulation. Fertil Steril 80(suppl 2): P747 - P754

Hess V, A'Hern R, Nasiri N, King DM, Blake PR, Barton DPJ, Shepherd JH, Ind T, Bridges J, Harrington K, Kaye SB, Gore ME (2004) Mucinous epithelial ovarian cancer: a separate entity requiring specific treatment. J Clin Oncol 22: $1040-1044$

Hinoi T, Lucas PC, Kuick R, Hanash S, Cho KR, Fearon ER (2002) CDX2 regulates liver intestine-cadherin expression in normal and malignant colon epithelium and intestinal metaplasia. Gastroenterol 123: $1565-1577$

Huflejt ME, Leffler H (2004) Galectin-4 in normal tissues and cancer. Glyconjugate J 20: $247-255$ 
Ilantzis C, DeMarte L, Screaton RA, Stanners CP (2002) Deregulated expression of the human tumor maker CEA and CEA family member CEACAM6 disrupts tissue architecture and blocks colonocyte differentiation. Neoplasia 4: $151-163$

Lee KR, Young RH (2003) The distinction between primary and metastatic mucinous carcinomas of the ovary: gross and histologic findings in 50 cases. Am J Surg Pathol 27: $281-292$

Lönnstedt I, Speed TP (2002) Replicated microarray data. Statist Sinica 12: $31-46$

Maggiora P, Lorenzato A, Fracchioli S, Costa B, Castagnaro M, Arisio R, Katsaros D, Massobrio M, Comoglio PM, Flavia di Renzo M (2003) The RON and MET oncogenes are co-expressed in human ovarian carcinomas and cooperate in activating invasiveness. Exp Cell Res 288: $382-389$

Maihle NJ, Baron AT, Barrette BA, Boardman CH, Christensen TA, Cora EM, Faupel-Badger JM, Greenwood T, Juneja SC, Lafky JM, Lee H, Reiter JL, Podratz KC (2002) EGF/ErbB receptor family in ovarian cancer. Cancer Treat Res 107: 247-258

Marquez RT, Baggerly KA, Patterson AP, Liu J, Broaddus R, Frumovitz M, Atkinson EN, Smith DI, Hartmann L, Fishman D, Berchuck A, Whitaker R, Gershenson DM, Mills GB, Bast Jr RC, Lu KH (2005) Patterns of gene expression in different histotypes of epithelial ovarian cancer correlate with those in normal fallopian tube, endometrium, and colon. Clin Cancer Res 11: 6116-6126

McCluggage WG (2000) Recent advances in immunohistochemistry in the diagnosis of ovarian neoplasms. J Clin Pathol 53: 327-334

Ono K, Tanaka T, Tsunoda T, Kitahara O, Kihara C, Okamoto A, Ochiai K, Takagi T, Nakamura Y (2000) Identification by cDNA microarray of genes involved in ovarian carcinogenesis. Cancer Res 60: 5007-5011

Platzer P, Upender MB, Wilson K, Willis J, Lutterbaugh J, Nosrati A, Willson JKV, Mack D, Ried T, Markowitz S (2002) Silence of chromosomal amplifications in colon cancer. Cancer Res 62: 1134-1138

Rapkiewicz AV, Espina V, Petricoin III EF, Liotta LA (2004) Biomarkers of ovarian tumours. Eur J Cancer 40: 2604-2612

Riopel MA, Ronnett BM, Kurman RJ (1999) Evaluation of diagnostic criteria and behavior of ovarian intestinal-type mucinous tumors: atypical proliferative (borderline) tumors and intraepithelial, microinvasive, invasive, and metastatic carcinomas. Am J Surg Pathol 23: $617-635$

Ronnett BM, Kajdacsy-Balla A, Gilks CB, Merino MJ, Silva E, Werness BA, Young RH (2004) Mucinous borderline ovarian tumors: points of general agreement and persistent controversies regarding nomenclature, diagnostic criteria, and behaviour. Hum Pathol 35: 949-960

Scanlan MJ, Chen Y-T, Williamson B, Gure AO, Stockert E, Gordan JD, Tureci O, Sahin U, Pfreundschuh M, Old LJ (1998) Characterization of human colon cancer antigens recognised by autologous antibodies. Int $J$ Cancer 76: $652-658$

Scholzel S, Zimmermann W, Schwarzkopf G, Grunert F, Rogaczewski B, Thompson J (2000) Carcinoembryonic antigen family members CEACAM6 and CEACAM7 are differentially expressed in normal tissues and oppositely deregulated in hyperplastic colorectal polyps and early adenomas. Am J Pathol 156: 595-605

Schwartz DR, Kardia SLR, Shedden KA, Kuick R, Michailidis G, Taylor JMG, Misek DE, Wu R, Zhai Y, Darrah DM, Reed H, Ellenson LH, Giordano TJ, Fearon ER, Hanash SM, Cho KR (2002) Gene expression in ovarian cancer reflects both morphology and biological behavior, distinguishing clear cell from other poor-prognosis ovarian carcinomas. Cancer Res 62: $4722-4729$

Segara D, Biankin AV, Kench JG, Langusch CC, Dawson AC, Skalicky DA, Gotley DC, Coleman MJ, Sutherland RL, Henshall SM (2005) Expression of HOXB2, a retinoic acid signaling target in pancreatic cancer and pancreatic intraepithelial neoplasia. Clin Cancer Res 11: $3587-3596$

Seidman JD, Horkayne-Szakaly I, Haiba M, Boice CR, Kurman RJ, Ronnett BM (2004) The histologic type and stage distribution of ovarian carcinomas of surface epithelial origin. Int J Gynecol Pathol 23: $41-44$

Seidman JD, Kurman RJ, Ronnett BM (2003) Primary and metatastic mucinous adenocarcinomas in the ovaries: incidence in routine practice with a new approach to improve intraoperative diagnosis. Am J Surg Pathol 27: $985-993$

Sherman ME, Mink PJ, Curtis R, Cote TR, Brooks S, Hartge P, Devesa S (2004) Survival among women with borderline ovarian tumor and ovarian carcinoma: a population-based analysis. Cancer 100: 1045-1052

Shih I-M, Kurman RJ (2004) Ovarian tumorigenesis: a proposed model based on morphological and molecular genetic analysis. Am J Pathol 164: $1511-1518$

Smyth GK (2004) Linear models and empirical Bayes methods for assessing differential expression in microarray experiments. Stat Appl Genet Mol Biol 3, Article 3

Takamura M, Ichida T, Matsuda Y, Kobayashi M, Yamagiwa S, Genda T, Shioji K, Hashimoto S, Nomoto M, Hatakeyama K, Ajioka Y, Sakamoto M, Hirohashi S, Aoyagi Y (2004) Reduced expression of liver-intestine cadherin is associated with progression and lymph node metastasis of human colorectal carcinoma. Cancer Lett 212: 253 - 259

Wang M-H, Wang D, Chen Y-Q (2003) Oncogenic and invasive potentials of human macrophage-stimulating protein receptor, the RON receptor tyrosine kinase. Carcinogenesis 23: $1291-1300$

Wright MD, Ni J, Rudy GB (2000) The L6 membrane proteins - a new fourtransmembrane superfamily. Prot Sci 9: 1594-1600 